\title{
Drowsy Driver Detection System Using Eye Blink Patterns
}

\author{
Taner Danisman, Ian Marius Bilasco, Chabane Djeraba, Nacim Ihaddadene \\ LIFL UMR CNRS 8022, Université Lille 1 \& Telecom Lille 1, Villeneuve d'Ascq 59655, Lille, France \\ Email: \{taner.danisman, marius.bilasco, chabane.djeraba, nacim.ihaddadene\}@lifl.fr
}

\begin{abstract}
This paper presents an automatic drowsy driver monitoring and accident prevention system that is based on monitoring the changes in the eye blink duration. Our proposed method detects visual changes in eye locations using the proposed horizontal symmetry feature of the eyes. Our new method detects eye blinks via a standard webcam in real-time at $110 \mathrm{fps}$ for a $320 \times 240$ resolution. Experimental results in the JZU [3] eye-blink database showed that the proposed system detects eye blinks with a $94 \%$ accuracy with a $1 \%$ false positive rate.
\end{abstract}

Keywords: Eye blink detection, eye symmetry, drowsiness detection.

\section{INTRODUCTION}

According to the latest report of the American National Highway Traffic Safety Administration (NHTSA) [1], the most influential factor in the occurrence of fatal single-vehicle runoff-road crashes is the driver performance-related factor: falling asleep, followed by alcohol use and vehicle speed. In addition $3.6 \%$ of fatal crashes are associated with the driver's drowsiness and fatigue [2].Over the last decade, an increased amount of effort and technology have been developed to prevent and reduce the effect of human related crashes (e.g. airbags, ABS, park sensors). A new technology called "Drowsy Driver Detection System" (DDDS) has been developed by major vehicle companies including Mercedes-Benz, Volvo, Saab, Nissan, and Hyundai which detect the fatigue state of the driver to prevent possible accidents. On the other hand, these studies have limitations in terms of the use of expensive hardware (e.g. infrared cameras) and the level of automaticity.

In this study, we present a low cost and fully automatic solution for handling the drowsy driver detection problem. Our system uses a standard webcam and detects the pattern of long duration eye-lid closures. The eye blink duration is the time spent while upper and lower eye-lids are connected. The pattern indicates a potential drowsiness prior to the driver falling asleep and then alerts the driver by voice messages. An operation flow diagram of the proposed system is shown in Figure 1.

A brief description of the existing approaches is presented in Section 2. Our methodology, including face detection, eye detection, eye blink detection and drowsiness detection, is described in Section 3. The experimental results on JZU Eyeblink database [3] are presented in Section 4. Finally, Section 5 concludes the paper and resumes the benefits of our solution.

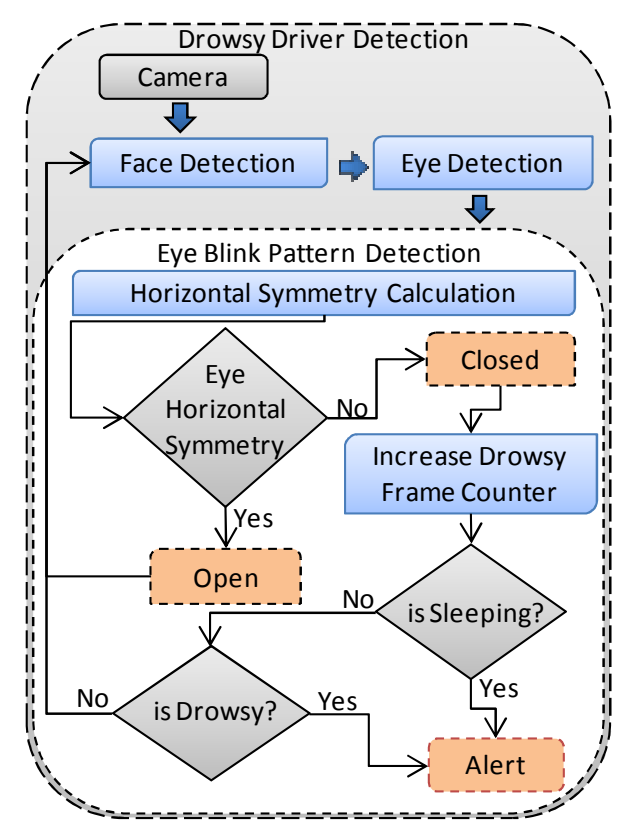

Figure 1. Drowsy driver detection flow diagram

\section{EXISTING APPROACHES}

DDDS has received a great deal of attention in both psychological and computer vision domains. We can divide the different DDDS approaches into two main categories.

The first category is related to physiological studies. It includes eye-lid closure, electrooculogram (EOG) [7] and electroencephalogram (EEG) [4], [5], [6]. Although the EOG provides good results, it is not suitable for a real driving environment because of the attached electrodes on the driver's face.

The second category is focused on the driving characteristics and unusual behavior of the vehicle (e.g. Volvo driver alert system) such as the use of reaction time of the driver and the steering wheel movements [8],[9],[23]. Among other methods, the eye-blink duration is the most reliable parameter for the detection of the drowsiness level [10].

Sukno, Pavani and Butakoffand et al. [12] detected eyeblinks in a given video sequence from a standard camera using Active Shape Models with the Invariant Optimal Features using 98 landmarks. They used the average distance between vertically corresponding landmarks represented by 8 landmarks 
per eye. Their study on Av@Car [22] database showed that the average blink duration is approximately $310 \pm 7.3 \mathrm{~ms}$. They achieved a $99.5 \%$ accuracy for open eye frames and $80.5 \%$ for closed eye frames. However, the use of 98 point Active Shape model is a time consuming task and it is not suitable for realtime applications where the minimum blink duration is $75 \mathrm{~ms}$ [13].

Morris, Blenkhorn and Zaidi [14] used a variance map for the real-time detection of eye blinks. But the performance of this approach strictly depends on head movements. Others used the optical flow [15], template matching [16] and deformable templates [12], [17].

The two main problems of the previous approaches are the high computational time and the initialization procedure. In this paper, we present a real-time approach that detects visual changes in eye locations using the horizontal symmetry feature of the eyes which does not require any initialization procedure. In addition, the proposed system can detect the state of the eye via a single frame without requiring additional frames.

\section{METHODOLOGY}

Our DDDS receives an input from a color video camera attached in front of the driver and processes the grabbed frames for the drowsiness detection. The detector system is composed of a video camera and software that regularly checks the eye of the driver to detect the eye blink duration.

We start with the detection of the face using the Viola Jones [18] face detector available in the OpenCV library. Then, we used the neural network-based eye detector [19] available in the STASM [20] library to locate the positions of the pupils. The STASM is a variation of the Active Shape Model of Coote's implementation [21]. We derived only the Rowley's eye detection code for real-time speed constraints from the STASM library which is a group of neural networks that provides eye positions.

After the eye detection, we estimate the orientation of the face using the vertical positions of both eyes. If they are not in the same position, we compute the angle between these two pupil points. Then, we correct the orientation of the face by rotating the whole frame in the opposite direction. The origin of the rotation is the face center. In this way, we eliminate the left and right rolling effect of the face up to \pm 25 degree.

Finally, we extract a rectangular area from the pupil area of the eye as seen in Figure 2. The width and height of the region of interest are respectively set to 0.5 and 0.16 of Inter Pupillary Distance (IPD) and resized to $20 \times 15$ for normalization.

In case of missing eye detection, we used the previous eye positions with respect to the detected face which provides the absolute position of the eyes. If only one eye is missing, then the position is computed considering both the current $\mathrm{dx}$ and dy difference of the detected eye with respect to its location in the previous frame. After that, displacement values the $\mathrm{dx}$ and $\mathrm{dy}$ values are added to the previous location of missed eye location.

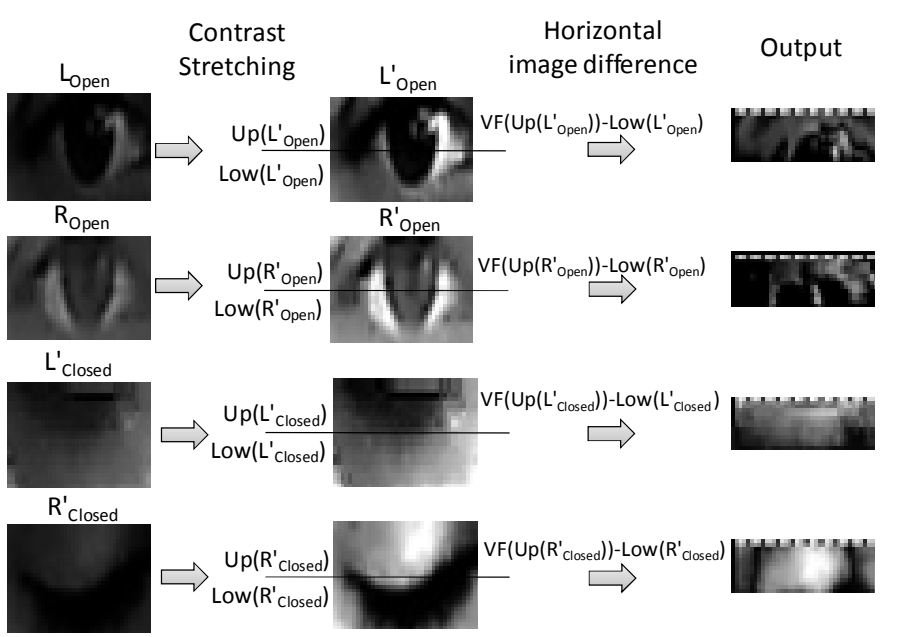

Figure 2. Eye blink detection. LOpen, ROpen, LClosed and RClosed are open and closed eye samples for the left and right eye respectively. $U p(X)$ and Low $(X)$ are the upper and lower halves of image $X$. $\operatorname{VF}(X)$ is the vertically flipped image of $\mathrm{X}$. The contrast in the output image is further enhanced for visualization purposes.

\section{A. Eye Blink Detection}

In order to detect different eye patterns (Open and closed), we first perform a contrast stretching using (1), (2) and (3).

$$
\begin{gathered}
\mathrm{I}(\mathrm{x}, \mathrm{y})=\operatorname{pow}\left(\frac{\mathrm{I}(\mathrm{x}, \mathrm{y})-\operatorname{low}_{\text {in }}}{\operatorname{err}_{\text {in }}}, \gamma\right) * \mathrm{err}_{\text {out }}+\operatorname{low}_{\text {out }} \\
\mathrm{err}_{\text {in }}=\text { high }_{\text {in }}-\operatorname{low}_{\text {in }} \\
\text { err }_{\text {out }}=\text { high }_{\text {out }- \text { low }_{\text {out }} .}
\end{gathered}
$$

Then, we horizontally divided the region of interest into two halves (Upper and Lower) with respect to the line passing through the center of the eye pupil as seen in Figure 2. Because of the circular shape of the eye, an open eye pattern has a horizontal symmetry property, whereas a closed eye does not have this property. Therefore, we proposed to use symmetry property as a discrimination function for open and closed eyes. We used (4), (5) and (6) to find the horizontal symmetry of the eye represented by $I_{\text {dif }}$ where $I^{\prime}$ is the contrast stretched and normalized image $\operatorname{VF}\left(\operatorname{Up}\left(I^{\prime}\right)\right)$ is the vertically flipped upper half of the image and Low $\left(\mathrm{I}^{\prime}\right)$ represents the lower half of the image. $I_{\text {sum }}$ is the cumulative sum value of the $I_{\text {dif }}$ image and $I_{\text {state }}$ is the detected status of the eye considering the $I_{\text {sum }}$ value.

$$
\begin{aligned}
& I_{\text {dif }}=V F\left(U p\left(I^{\prime}\right)\right)-\operatorname{Low}\left(\mathrm{I}^{\prime}\right) . \\
& I_{\text {sum }}=\sum_{i=0, j=0}^{\text {width,height }} I_{\text {dif }}(i, j) \\
& I_{\text {state }}=\left\{\begin{array}{cc}
\text { Open } & I_{\text {sum }}<T \\
\text { Closed } & I_{\text {sum }} \geq T
\end{array}\right\}
\end{aligned}
$$

Value $T$ represents the threshold limit for a closed eye. In case there is no symmetry feature, the values in the difference image $\mathrm{I}_{\text {dif }}$ will be higher, and higher values of the cumulative sum of the difference image $I_{\text {dif }}$ create a brighter output image 
which corresponds to a closed eye state. Considering our own experiments, we selected to use $T=12.000$ which gives the lowest false positive rate.

\section{B. Drowsiness Detection}

We define three states for the driver drowsiness as seen in TABLE I. Considering the Caffier's study [13], the typical eye blink duration is less than $400 \mathrm{~ms}$ on average and $75 \mathrm{~ms}$ for minimum. For this reason, we used $T_{\text {Drowsy }}=400 \mathrm{~ms}$ and $\mathrm{T}_{\text {Sleeping }}=800 \mathrm{~ms}$.

TABLE I.

THREE LEVEL FOR DROWSINESS.

\begin{tabular}{cc}
\hline Drowsiness Level & Description \\
\hline Awake & Blink durations $<\mathrm{T}_{\text {Drowsy. }}$ \\
\hline Drowsy & $\begin{array}{c}\text { Blink durations }>\mathrm{T}_{\text {Drowsy }} \text { and Blink } \\
\text { durations }<\mathrm{T}_{\text {Sleeping }}\end{array}$ \\
\hline Sleeping & Blink durations $>\mathrm{T}_{\text {Sleeping }}$ \\
\hline
\end{tabular}

Drowsiness detection is directly connected to the eye blink detection component. The timing of eye closed events is compared with the threshold values. Considering a car at $90 \mathrm{~km} / \mathrm{h}$, the $\mathrm{T}_{\text {Drowsy }}$ and $\mathrm{T}_{\text {Sleeping }}$ values corresponds to $10 \mathrm{~m}$ and $20 \mathrm{~m}$ of distances which gives enough time for the system to give an alarm signal to the driver.

\section{EXPERIMENTAL RESULTS}

In literature, the PERCLOS (PERcentage of eye CLOSure) [11] value has been used as drowsiness metric which shows the percentage of time in a minute that the eyes are $80 \%$ closed. In addition, we manually marked the frames that eyes are at least $80 \%$ closed as seen in Figure 3.

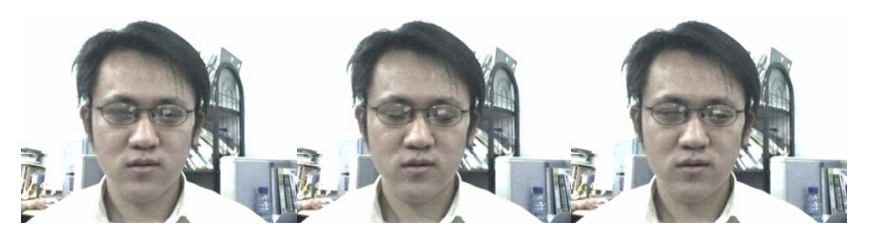

Figure 3. JZU database sample for closed eye (Video: 000001M_FBN.avi, Frames: 35, 39 and 42) All frames between the $35^{\text {th }}$ and $42^{\text {nd }}$ frames are labeled as closed eye frame since both eyes are at least $80 \%$ closed.

According to our manual annotation results, the average eye blink duration is $205 \mathrm{~ms}$ or 6.17 frames for $30 \mathrm{fps}$ videos. We identified 272 eye blinks in 80 videos in the JZU database. Ground truth data for eye blink were obtained manually observing 11,750 frames.

Using the proposed method, we achieved a $94 \%$ accuracy in the JZU database using all 80 videos, 40 of them include users wearing glasses. The videos include both static and moving faces as well as dynamic backgrounds with different lighting conditions. TABLE II. Shows a summary of the results obtained in the JZU database.

TABLE II. EXPERIMENTAL RESULTS IN TERMS OF ACCURACY, PRECISION AND RECALL OBTAINED IN THE JZU DATABSE.

\begin{tabular}{lr}
\hline Accuracy & $94.8 \%$ \\
\hline Precision & $90.7 \%$ \\
\hline Recall & $71.4 \%$ \\
\hline False Positive Rate (FPR) & $1 \%$ \\
\hline
\end{tabular}

\section{CONCLUSION}

We proposed a new method to detect eye blinks using the symmetry property. The proposed system is independent from the head movements as it works within the same frame. Therefore, it has an advantage over the other systems that use statistical information from the past frames. In addition, it runs at a 110fps rate on Intel Xeon $2.9 \mathrm{GHz}$ CPU for a $320 \times 240$ resolution which is acceptable for real-time scenarios and leaves time for other tasks. Unfortunately, no common database exists for comparing our results for drowsiness; therefore we only give the results for eye-blink detection. In addition, we planned to work on the Av@Car database for further comparison of our approach.

The proposed system detects eye blinks with a 94\% accuracy and a $1 \%$ false positive rate. Our experiments showed that the proposed system produces fast and accurate results for the detection of drowsiness. According to the real world experiments, the most important factors that affect the performance of our method are the presence of glasses and high illumination changes. Indeed, the presences of glasses affect the core components of the system including face detection, eye detection and symmetry calculation.

\section{ACKNOWLEDGMENT}

This work has been supported by the MIDAS (Multimodal Interfaces for Disabled and Ageing Society) ITEA 2 - 07008 project.

\section{REFERENCES}

[1] C. Liu and R. Subramanian, "Factors Related to Fatal Single-Vehicle Run-Off-Road Crashes," U.S. Department of Transportation, American National Highway Traffic Safety Administration, DOT HS 811 232, Washington, D.C., November, 2009.

[2] R.R. Knipling and J.S. Wang, "Crashes and Fatalities Related to Driver Drowsiness, Fatigue," National Highway Traffic Safety Administration, Washington, D.C., Information Management Consultants Inc., McLean, VA., 1994.

[3] G. Pan, L. Sun, Z. Wu and S. Lao, "Eyeblink-based Anti-spoofing in Face Recognition from a Generic Webcamera," The 11th IEEE International Conference on Computer Vision (ICCV'07), Rio de Janeiro, Brazil, October, 2007.

[4] J. Santamaria and K. Chiappa, "The EEG of drowsiness in normal adults," Journal of Clinical Neurophysiology, vol. 4, no. 4, pp. 327-382, 1987.

[5] R.P. Nikhil, C. Chien-Yao, L.W. Ko, C.F. Chao, T.P. Jung, S.F. Liang and C.T. Lin, "EEG-based Subject-and Session-independent Drowsiness Detection: An Unsupervised Approach," EURASIP Journal on Advances in Signal Processing, ISSN: 1110-8657, January 2008.

[6] A. Vuckovic, V. Radivojevic, A.C.N. Chen and D. Popovic, "Automatic recognition of alertness and drowsiness from EEG by an artificial neural network," Medical Engineering \& Physics, vol. 24, no. 5, pp. 349, 2002.

[7] B. James, H. Sharabaty and D. Esteve, "Automatic EOG analysis: A first step toward automatic drowsiness scoring during wake-sleep transitions," Somnologie, vol. 12, pp. 227-232, 2008.

[8] J.H. Yang, Z.H. Mao, L. Tijerina, T. Pilutti, J.F. Coughlin and E. Feron, "Detection of driver fatigue caused by sleep deprivation," IEEE Transactions on Systems, Man and Cybernetics, vol. 39, July 2009.

[9] C.C. Liu, S.G. Hosking and M.G. Lenné, "Predicting driver drowsiness using vehicle measures: Recent insights and future challenges," Journal of Safety Research ,vol. 40, no. 4, pp. 239-245, August 2009.

[10] D. Dinges, "PERCLOS: A valid psychophysiological measure of alertness as assessed by psychomotor vigilance indianapolis," Federal 
Highway Administration, Office of Motor Carriers, Technical report, MCRT-98-006, 1998

[11] W.W. Wierwille, L.A. Ellsworth, S.S. Wreggit, R.J. Fairbanks and C.L. Kirn, "Research on vehicle based driver status/performance monitoring: development, validation and refinement of algorithms for detection of driver drowsiness," National Highway Traffic Safety Administration, Technical report, DOT HS 808 247, 1994.

[12] F.M. Sukno, S.K. Pavani, C. Butakoffand and A.F. Frangi, "Automatic Assessment of Eye Blinking Patterns through Statistical Shape Models," ICVS 2009, LNCS 5815, Springer-Verlag Berlin Heidelberg, pp. 33-42, 2009.

[13] P.P. Caffier, U. Erdmann and P. Ullsperger, "The spontaneous eye-blink as sleepiness indicator in patients with obstructive sleep apnoea syndrome - a pilot study," Sleep Medicine, vol 6., no. 2, pp. 155-162, 2005.

[14] T. Morris, P. Blenkhorn and F. Zaidi, "Blink detection for real-time eye tracking," Journal of Network and Computer Applications, vol. 25, no. 2, pp. 129-143, April 2002.

[15] M. Divjak and H. Bischof, "Eye blink based fatigue detection for prevention of Computer Vision Syndrome," MVA2009 IAPR Conference on Machine Vision Applications, Yokohama, Japan, May 2009.

[16] M. Chau and M. Betke, "Real Time Eye Tracking and Blink Detection with USB Cameras," Boston University Computer Science Technical Report No. 2005-12, 2005.
[17] L. Wang, X. Ding, C. Fang and C. Liu, "Eye blink detection based on eye contour extraction," Proceedings of SPIE, vol. 7245, 72450R, 2009.

[18] P. Viola and M. Jones, "Rapid object detection using a boosted cascade of simple features," International Conference on Computer Vision and Pattern Recognition, CVPR 2001.

[19] H.A. Rowley, S. Baluja and T. Kanade, "Neural Network-Based Face Detection," IEEE Transactions on Pattern Analysis and Machine Intelligence, vol. 20, pp. 23-38, http://vasc.ri.cmu.edu/NNFaceDetector, 1998.

[20] S. Milborrow and F. Nicolls, "Locating facial features with an extended active shape model," In D. Forsyth, P. Torr and A. Zisserman (eds.) ECCV 2008, Part IV. LNCS, vol. 5305, 504--513. Springer-Verlag, Heidelberg, 2008

[21] T.F. Cootes, G.J. Edwards and C.J. Taylor, "Active appearance models," In H.Burkhardt and B. Neumann, editors, 5th European Conference on Computer Vision, vol. 2, 484--498. Springer, Berlin, 1998.

[22] A. Ortega, F. Sukno, E. Lleida, A. Frangi, A. Miguel, L. Buera and E. Zacur, "AV@CAR: A spanish multichannel multimodal corpus for in-vehicle automatic audio-visual speech recognition," In M.T. Lino, M.F. Xavier, F. Ferreira, R. Costa and R. Silvia, editors, IV International Conference on Language Resources and Evaluation, vol. 3, pp. 763-767, European Language Resources Association, May 2004.

[23] E. Vural, M. Cetin, G. Littlewort, M. Bartlett and J. Movellan "Drowsy Driver Detection Through Facial Movement Analysis," Lecture Notes in Computer Science, vol. 4796, pp. 6-18, 2007. 